\title{
SCHELLING CONTRA JACOBI \\ LA POSIBILIDAD DE UN SISTEMA \\ DE LA LIBERTAD
}

\author{
HERNÁN GUERRERO TRONCOSO \\ Universidad Católica del Maule
}

\begin{abstract}
RESUMEN: Este artículo intenta mostrar cómo el examen del supuesto panteísmo del sistema de Spinoza, que abre las Investigaciones filosóficas sobre la esencia de la libertad humana de Schelling, presenta no solo su propia posición ante la polémica del panteísmo, sino que también implica una discusión con el pensamiento moderno, en particular en lo que se refiere al lugar, función e implicancias de la noción de sistema y su relación con la libertad. Una exposición del análisis crítico de Jacobi de la historia de la filosofía occidental - en el que afirma que el sistema no es compatible con el conocimiento de lo incondicionado- permitirá iluminar los términos en los que se plantea este diálogo del pensador de Leonberg con la tradición.
\end{abstract}

PALABRAS CLAVE: Idealismo alemán, Ontología, Panteísmo, Spinoza, Voluntad.

\section{Schelling against Jacobi The Possibility of a System of Freedom}

\begin{abstract}
The intention of this article is to show how the examination of the alleged pantheism of Spinozas' system, which opens the Philosophical Inquiries into the Essence of Human Freedom of Schelling, presents not only his own stand concerning the Pantheism controversy, but also implies a discussion with Modern thought, particularly regarding the place, function and implications of the notion of system, and its relation to freedom. An exposition of Jacobi's critical analysis of the history of Western Philosophy - where he asserts that the system is incompatible with the knowledge of the unconditioned- will illustrate the terms of this dialogue of the philosopher from Leonberg with the tradition.
\end{abstract}

KEY WORDS: German Idealism, Ontology, Pantheism, Spinoza, Will.

\section{INTRODUCCIÓN}

Entre las distintas cuestiones que dan que pensar en la Época Moderna, la pregunta por Dios puede ser sin duda considerada como uno de los problemas fundamentales, en la medida en que en ella se deciden asuntos de orden ontológico y gnoseológico de los cuales depende la comprensión de la realidad en general y del lugar que ocupa el hombre en medio de ella. Esto se hace aún más evidente si se considera que, en último término, el rol que juega Dios en el despliegue de la realidad es sustancialmente el mismo que tuvo en la Edad Media - en particular durante la Escolástica-, a pesar de que entre ambas épocas es posible apreciar una diferencia radical en la comprensión del concepto de ser, y en consecuencia de los términos en que se concibe la relación entre el mundo y Dios. Así, por ejemplo, cuando un Doctor de la Escuela como el beato Juan Duns Scoto interpreta la doctrina agustiniana de la iluminación divina, no se limita a examinar sus consecuencias gnoseológicas, sino que se preocupa de 
mostrar cuáles son los términos de la relación que se da entre Dios y las criaturas, según la cual Dios no solo ilumina al hombre para que pueda conocer la realidad, sino que, en la medida en que es causa y fundamento de la realidad, y Él mismo es la realidad más alta, es causa objetiva de todo conocimiento, aún cuando no lo infunda en el intelecto humano mediante una iluminación especial (Duns Scoto, 1954: 123-172; Brown, 1976: 99-111; íd., 1984: 140-180).

En la Modernidad, en cambio, los términos se comprenden de manera inversa. En efecto, por una parte se afirma —o más bien se postula-que Dios es el fundamento último de la realidad, pero al mismo tiempo su existencia y realidad se conciben según el modo en que los objetos de la experiencia existen y son reales, ya que dicho modo es la medida según la cual nuestro entendimiento es capaz de conocer a ciencia cierta. Así, la realidad de un objeto depende en último término de que su representación tenga lugar en una conciencia, la cual a su vez debe ser consciente de la presencia actual de dicho objeto en ella (cf. Descartes, 1982: 6-12; íd., 1983: 34-52). En este sentido, se puede afirmar que, si los medievales intentan mostrar que la realidad del ámbito de las criaturas encuentra su fundamento en su remisión al Creador, los modernos consideran como real solo aquello que se constituye como objeto de manera cierta ante la conciencia, y a partir de esa comprensión de lo real pretenden concebir a Dios en cuanto aquello que contiene en sí toda la realidad (cf. Kant, 1998: 668-676).

Esto deja en evidencia otra diferencia fundamental entre ambas épocas, y que se refiere al conocimiento que puede tener el hombre de Dios. Para los medievales, ese conocimiento se basa en último término en una doctrina revelada. En este sentido, como sostiene Duns Scoto (1950: 4), «los filósofos afirman el carácter perfecto de la naturaleza - y con ello la suficiencia del conocimiento natural-, y niegan que haya una perfección sobrenatural. Por el contrario, los teólogos reconocen el carácter carente de la naturaleza, la necesidad de la gracia y la perfección sobrenatural». Esto significa que el conocimiento de las criaturas, si bien constituye un modo en que el hombre es capaz de advertir en esta vida la presencia de Dios en el mundo, no es suficiente por sí solo para hacer que nuestra razón alcance un conocimiento adecuado de Él. Es aquí que la Revelación aparece como una guía para reconocer en qué consiste el Dios que se manifiesta en las criaturas, y que según el Doctor Sutil puede ser concebido como un ser infinito en acto. (Duns Scoto, 1950: 2; ibíd., 38-40; íd., 1954: 40-41; Guerrero Troncoso, 2016: 421-438).

Los modernos, por su parte, que no recurren a la Sagrada Escritura como fuente de su pensamiento, se vieron igualmente obligados en algún momento a reconocer que la razón por sí sola no es capaz de concluir la existencia de Dios de manera puramente especulativa, ya que la existencia no constituye una condición de posibilidad de la experiencia, sino que es más bien el punto de partida y el primer presupuesto del conocimiento, y solo los objetos de la experiencia poseen las condiciones necesarias para poner en evidencia a ciencia cierta su existencia, lo cual ocurre solo a posteriori (cf. Kant, 1998: 253-256). Sin embargo, encontraron en la voluntad, y en particular en la noción de un bien absoluto e infinito, un medio para al menos postular la existencia de Dios 
y, con ello, para reconducir la actividad libre y creadora de Dios al ámbito de la experiencia, en la medida en que el actuar del hombre se lleva a cabo a partir de una buena voluntad (Kant, 1998: 832-859).

Es en vistas de esta comparación entre lo que reúne al Medioevo y a la Modernidad, y que a la vez los distingue, que el pasaje con el que F. W. J. Schelling concluye su análisis del panteísmo - justo antes de comenzar el examen de la esencia de la libertad humana y los objetos que están relacionados con ellaresulta particularmente iluminador. En efecto, más que concluir una discusión simplemente doctrinal, las siguientes líneas dan una visión crítica de los rasgos más propios de la época moderna:

El concepto fundamental de Spinoza, espiritualizado por el principio del Idealismo (y transformado en un punto esencial), alcanzó en un modo superior de consideración de la naturaleza, y en la reconocida unidad de lo dinámico con lo anímico y lo espiritual, una base viva, a partir de la cual surgió una Filosofía de la Naturaleza que podría subsistir por sí misma en tanto que mera física, pero que en relación con la totalidad de la filosofía fue considerada en todo tiempo como una sola parte de ella, en concreto, como la parte real, que solo entonces se torna capaz de elevarse hasta alcanzar el auténtico sistema de razón, añadiéndole la parte ideal, en la que reina la libertad. Se afirma que en ella (en la libertad) reside el último acto potenciador, gracias al cual toda naturaleza se transfigura en sensación, en inteligencia y, finalmente, en voluntad. - En la más alta y última instancia, no hay otro ser sino querer. Querer es el ser originario [Ursein] y solo en él concuerdan todos los predicados de este último: ausencia de fundamento, eternidad, independencia respecto al tiempo, autoafirmación. Toda la filosofía pugna solo por esto, por encontrar esta expresión suprema (Schelling, 2011: 23).

En la parte central de este párrafo, Schelling menciona el «auténtico sistema de razón». Dicha mención podría pasar desapercibida, si no fuera porque toda la discusión anterior, en la que se ocupa del panteísmo, constituye una respuesta a la afirmación que resume el problema fundamental de la controversia conocida como la "polémica del espinosismo", a saber, que "el único sistema posible de la razón es el panteísmo, pero este es irremediablemente fatalismo» (Schelling, 2011: 11). Con esto, todos los elementos constitutivos de la Modernidad están presentes, ya que en esa época la forma que adopta el conocimiento científico en general, y la filosofía en particular, es el sistema (cf. Hegel, 1998: 6).

La concurrencia de tales nociones — panteísmo, libertad y sistema- en un mismo párrafo no tiene nada de antojadiza, y deja en evidencia que el vínculo que las reúne o las distingue no posee un carácter meramente conceptual - como si de diera una identificación o una exclusión formal entre ellas, en el sentido de que no se puede concebir el sistema sin que se convierta en determinismo, fatalismo o panteísmo, o que sea totalmente incompatible con la libertad - sino que posee más bien un carácter histórico. En efecto, del examen crítico que precede a este fragmento queda claro que el vínculo entre dichos elementos proviene de la controversia que suscitó una meditación en torno al 
panteísmo, y la consiguiente reflexión en torno a dicha cuestión determinó el sentido en el que los más grandes pensadores que surgieron durante esa polémica —en particular Hölderlin, Hegel y el mismo Schelling — entendieron cómo la filosofía se conforma esencialmente como sistema. En este sentido, la necesidad de aquel vínculo posee también un carácter histórico, y por ende contingente, de manera que el examen de su validez se debe llevar a cabo como una discusión con la tradición.

La tradición, en este caso, ya había sido interpretada en época de Schelling en más de una ocasión, pero hay un texto en particular que, junto con haber provocado un remezón en la intelectualidad de la época - e, indirectamente, incluso la muerte de uno de los involucrados (cf. Beiser, 1987: 61-75; Freudiger, 1993; Jakuszko, 2009: 174-176; Wulf, 2013: 129-130)—, dejó una profunda huella en los pensadores de finales del siglo xvIII. Dicho texto es el escrito Sobre la doctrina de Spinoza en cartas al Sr. Moses Mendelssohn, de F. H. Jacobi. En él, Jacobi reconstruye el pensamiento de Spinoza, al tiempo que formula sus críticas a partir de aquello que para él constituye el problema fundamental de la filosofía moderna - a saber, su carácter mecanicista, esto es, determinista, y por ende fatalista - dado que la forma que adopta el pensamiento moderno es precisamente el sistema. Para el filósofo de Düsseldorf, la consecuencia más grave del carácter mecanicista del sistema sería el hecho de que la libertad quedaría excluida de la filosofía (Kahlefeld, 2000: 30-35).

Precisamente, esa comprensión de la noción de sistema en general, y no solo del de Spinoza - de hecho, la crítica de Jacobi se extiende de igual manera a los filósofos cabalísticos y a Leibniz ${ }^{1}$ - fue lo que en último término movió a Schelling a examinar si el sistema necesariamente conduce al panteísmo, y si eso es incompatible con la libertad, o si se trata más bien de un problema de interpretación ${ }^{2}$. Ahora bien, para que quede en evidencia cuál es la cuestión que el examen del panteísmo de Schelling intenta responder, a continuación se expondrá brevemente el apéndice vII del escrito sobre Spinoza (Jacobi, 2000: 273-293; Jakuszko, 2009: 179-183), en el cual es el mismo Jacobi quien precisa el contexto y el alcance de su identificación de la noción de sistema con el panteísmo y el fatalismo. Esto permitirá que se entienda mejor hacia dónde

1 Cf. JACOBI, 2000: 118-124: «I. Spinozismus ist Atheismus. - II. Die Kabbalistische Philosophie, so viel davon der Untersuchung offen liegt, und nach ihren besten Kommentatoren, von Helmont dem Jüngeren, und Wachter, ist, als Philosophie, nicht anders, als unentwickelter, oder neu verworrener Spinozismus. - III. Die Leibniz-Wolffische Philosophie, ist nicht minder Fatalistisch, als die Spinozistische, und führt den unablässigen Forscher, zu den Grundsätzen der letzteren zurück. - IV. Jeder Weg der Demonstration geht in den Fatalismus aus. - V. Wir können nur Ähnlichkeiten demonstrieren, und jeder Erweis setzt etwas schon Erwiesenes zum voraus, wovon das Prinzipium Offenbarung ist. - VI. Das Element aller menschlichen Erkenntnis und Würksamkeit, ist Glaube»; FreUdiger, 1993: 45; JAKUszKo, 2009: 176-179.

2 Sin embargo, aquello que dio pie para que Schelling saliera en su propia defensa fue el escrito de Schlegel F. Sobre la lengua y la sabiduría de los Indios (1808), en el que critica duramente al filósofo de Leonberg; al respecto, cf. CARRASCo CONDE, 2011: 93-103. 
apunta el examen de los distintos tipos de panteísmo que identifica Schelling y la importancia histórica y especulativa de la aclaración de su propia posición al respecto.

\section{LAS OBJECIONES DE JACOBI AL ESPINOSISMO SON FUNDAMENTALMENTE OBJECIONES AL SISTEMA COMO FORMA DE LA FILOSOFÍA}

En la segunda edición de su escrito sobre Spinoza, Jacobi consideró necesario discutir algunas afirmaciones del texto original con mayor detención. Para ello, añadió ocho apéndices — nueve, si se cuenta el breve examen de la libertad que se halla después del prólogo ${ }^{3}$ - entre los cuales incluso se encuentra una sección casi completa del tratado De la causa, principio et uno de Giordano Bruno (cf. Jacobi, 2000: 195-220). Por su parte, en el apéndice vII, el filósofo de Düsseldorf examina algunas de las frases más polémicas de su escrito, que son las siguientes:

Pág. 61: «El Dios de Spinoza es el principio puro de la realidad actual de todo lo que es actualmente real, del ser en toda existencia, absolutamente sin individualidad, y completamente infinito. La unidad de este Dios radica en la identidad de lo indiscernible, y no excluye [que se dé] una especie de pluralidad» - Pág. 225: «Todo camino de la demostración termina en fatalismo». — Págs. 45 y 46: «Lessing se mantuvo [firme] en esto: él hubiera querido preguntar por todo de manera natural; y yo, [me mantuve firme en] que no puede haber una filosofía natural de lo sobrenatural, si bien ambos (lo natural y lo sobrenatural) estarían evidentemente a la mano» (Jacobi, 2000: 271).

En lo que viene a continuación, el filósofo de Düsseldorf pretende mostrar el contexto especulativo en el cual deben ser entendidas dichas afirmaciones. Dicho contexto, sin embargo, es intrínsecamente histórico. En efecto, Jacobi deja en claro que estas afirmaciones deben ser leídas a partir del modo en que interpreta las doctrinas filosóficas que se han dado a lo largo de la historia, tomando como clave de lectura la manera en que el hombre ha intentado responder el problema del origen del universo, esto es, el modo en que la razón humana ha determinado en qué consiste el primer principio de toda realidad. Así, en primer lugar, nuestro filósofo advierte una continuidad en las cuestiones que han llevado a pensar a los filósofos a lo largo de la historia y una regularidad en su manera de proceder. Con eso quedaría de manifiesto que el actuar de la filosofía sería reflejo de cómo opera la razón misma. De hecho, Jacobi considera que desde antiguo la tarea de los filósofos ha sido la de encontrar algo permanente

3 Cf. JACOBI, 2000: 160-161: «Eine neunte Beilage soll ihren Platz in diesem Vorrede erhalten, nachdem ich mir den Weg dazu die Beantwortung eines Vorwurfs gebahnt haben werde, der mir von Herrn Rehberg allein zweimal hintereinander, und ich glaube von mehreren gemacht worden ist». Jacobi presenta una suerte de examen de la posibilidad de la libertad humana inmediatamente después del prólogo (ibíd., 166-180). 
en la naturaleza, que se presenta sometida a un cambio perpetuo. Sin embargo, ya que no pudieron encontrar en los objetos eso permanente, prefirieron entonces considerar como objetivo y permanente aquello que el sentido común compone a partir de lo que le representa cada uno de los sentidos. De esta manera, el entendimiento, que ya no debía encontrar las cualidades ocultas de los objetos de la experiencia, terminó por considerar como principios fundamentales de la realidad las nociones empíricas de existencia y coexistencia, acción y reacción, espacio y movimiento, conciencia y pensamiento. Asimismo, puesto que a la base de todo conocimiento se encuentra una existencia viva, y tanto más vivo es un ser cuanto más variadas son las representaciones mediante las cuales se da a conocer, fue necesario que surgieran la abstracción y el lenguaje para reunir las variadas representaciones y darles realidad. De esta manera, se terminó originando un mundo de la razón, en donde cifras y palabras tomaron el lugar de las sustancias y sus fuerzas (cf. Jacobi, 2000: 272-274). Como consecuencia de esto, dice nuestro filósofo:

Nos apropiamos del universo en la medida en que lo despedazamos y creamos un mundo de imágenes, ideas y palabras, adecuado a nuestras capacidades y totalmente disímil al [mundo] real. Lo que creamos de esta manera lo entendemos - en la medida en que es nuestra creación-completamente; lo que no se deja crear de esta manera, no lo entendemos; nuestro entendimiento filosófico no se extiende más allá de su propio producto (Jacobi, 2000: 274).

Este fragmento deja en claro que, para Jacobi, el pensamiento que surgió en la Época Moderna, específicamente con Descartes, marcó un giro radical con respecto a la relación que tradicionalmente se había dado entre naturaleza y razón, de tal modo que desde entonces es la razón la que decide la realidad de la naturaleza. En este sentido, la realidad de todo cuanto se representa como perteneciente a la naturaleza depende del hecho de que la razón sea capaz de crear el objeto que reúne en sí aquellas distintas representaciones, esto es, de poner diferencias entre ellas, para luego darlas por superadas en el objeto percibido que las reúne en sí. Más aún, dicho giro coincidiría con el modo en que la razón misma despliega su actividad. Así, Jacobi concluye este párrafo afirmando que "[la acción de] percibir, reconocer y concebir, en relaciones ascendentes, constituye la entera totalidad de nuestra capacidad intelectual» (Jacobi, 2000: 274).

Pero esta revolución de la manera de concebir la relación entre naturaleza y razón tiene también un sentido histórico intrínseco, que se muestra decisivo para entender el vínculo entre sistema y determinismo. Jacobi observa que en el pensamiento moderno se da una remisión del ámbito de la cualidad —donde encontramos una multiplicidad infinita - al ámbito de la cantidad — donde en cambio tienen lugar solo algunas determinaciones fundamentales-, remisión con la cual alcanza su consumación el camino iniciado ya en la antigua Grecia por Leucipo y Demócrito y retomado por Descartes. Pues bien, aún cuando nuestro filósofo reconoce que a este modo de comprender la realidad se le deben agradecer los asombrosos avances de la ciencia natural moderna, también señala que dos grandes problemas quedaron sin solución, a saber, la 
imposibilidad de derivar a partir de las propiedades de la sustancia corporal una sustancia pensante, y la imposibilidad de procurar una existencia natural para el movimiento y sus modificaciones (cf. Jacobi, 2000: 274-275).

Es aquí que el panteísmo de Spinoza surge como un intento de solución de dichas cuestiones. Así, en lo que respecta a la relación entre la sustancia corporal y la sustancia pensante, dado que materia y forma ya no se consideran sustancias, sino modos presentes en una única sustancia, resulta que el cuerpo no puede ser concebido sin el pensamiento y este último tampoco puede serlo sin el cuerpo (Spinoza, 2010: 124-126; Wolfson, 1934b: 33-64). Esta primera afirmación permitiría resolver el segundo problema, el de la realidad del movimiento, postulando que todas las cosas individuales surgen de la única sustancia de manera natural, es decir, necesaria y mecánica, en virtud precisamente de la identificación entre materia y forma que tiene lugar por su presencia simultánea en la sustancia. Ahora bien, no obstante el hecho de que Jacobi valore positivamente que, a diferencia de otros filósofos, con esta solución Spinoza no trató de conciliar la noción de un caos originario o de un universo que se despliega de manera paulatina, con la noción de un principio natural que actúa eternamente - dos nociones que en el fondo se excluyen mutuamente-, no es posible sostener que Spinoza haya cumplido con su propósito de aclarar de manera natural la existencia de cosas finitas y sucesivas (Jacobi, 2000: 275276).

La crítica de Jacobi apunta fundamentalmente al hecho de que la afirmación de que existe una serie infinita y sucesiva de cosas individuales supone a su vez un tiempo eterno, esto es, una finitud infinita. Luego, dado que Spinoza mismo se habría dado cuenta de lo impropio de una suposición tal, se habría refugiado en similitudes matemáticas para demostrar la validez de su posición, para decir que la representación de un tiempo eterno es un engaño de nuestra imaginación. Sin embargo, el que se habría dejado engañar por su imaginación habría sido más bien Spinoza, según Jacobi, ya que la sucesión que representan las similitudes matemáticas no posee un carácter objetivo ni real, sino puramente subjetivo e ideal, e incluso ni siquiera ideal, si es que no se da una sucesión real en el sujeto. En este sentido, el gran error en que habría incurrido el filósofo holandés - y con él, según nuestro autor, varios otros filósofos de primer orden - sería el hecho de separar y volver a reunir lo subjetivo y lo objetivo, y de intercambiar arbitrariamente sus funciones en cuanto a cuál es la causa y cuál es el efecto (Jacobi, 2000: 276-277).

Ahora bien, Jacobi considera correcta la conclusión de Spinoza —que la actividad eterna e infinita de la materia debe ser un modo propio e inmediato de la sustancia, ya que el devenir no pudo haber sido engendrado-, e incluso sostiene que las bases para dicha conclusión habían sido sentadas ya en la Escolástica, con la noción de una creación perpetua. De hecho, mediante la determinación de Dios en cuanto natura naturans y de las demás cosas como naturae naturatae, Spinoza habría evitado varias de las dificultades que enfrentaban quienes proponían la doctrina de una creación perpetua, entre los que se encontraría el mismo Leibniz. Sin embargo, tanto en el caso de Spinoza 
como en el de los creacionistas sería posible advertir un error común, el que consistiría en que habrían confundido el concepto de fundamento con el de causa, y habrían convertido este último concepto — que posee un carácter más bien empírico- en una entidad lógica. Ahora bien, Jacobi no estima que sea inaceptable asociar los conceptos de causa y fundamento - tal como ocurre en el principio de razón suficiente-, siempre y cuando con ello no se pasen por alto sus diferencias (Jacobi, 2000: 278-283).

Mediante la reflexión anterior, Jacobi intenta reafirmar su posición, a saber, que no se puede explicar en absoluto de manera natural la existencia de un mundo constituido por una sucesión infinita de cosas finitas. En efecto, dicha existencia es incluso inconcebible. Este es el punto fundamental de la posición de nuestro filósofo, y por ende no se trataría simplemente del hecho de que algunas doctrinas a lo largo de la historia no hayan sido capaces de dar un concepto adecuado del principio de la sucesión de las cosas, sino que el principio mismo - ya sea que consista en una sucesión infinita, o bien en una inteligencia o en un organismo que preceda todos los organismos- es inconcebible, es decir, es imposible que sea aprehendido por nuestra razón de la misma manera en que ella comprende la realidad. Por lo tanto, el fracaso de estos sistemas radicaría en el hecho de que intentan convertir lo extra o sobrenatural en algo natural, y lo natural en algo sobrenatural. Jacobi concluye que:

Todo lo que la razón puede sacar mediante su [acción de] desglosar, vincular, juzgar, concluir y volver a concebir, son meras cosas de la naturaleza, y la razón misma pertenece — como [el] ser limitado [que es] — a estas cosas. Sin embargo, la naturaleza en su totalidad, la suma de todos los seres condicionados, no puede revelar al entendimiento que la examina más de lo que está contenido en ella, a saber, una existencia múltiple, transformaciones, un juego de formas, y jamás un comienzo EN ACTO, nunca un principio REAL de alguna existencia objetiva (Jacobi, 2000: 286).

La consecuencia más importante de esto es que, en la medida en que la razón intenta conocer lo incondicionado del mismo modo en que conoce la naturaleza - cuyo conocimiento es condicionado-, al parecer se encontraría en contradicción consigo misma. Jacobi afirma que la conciencia humana está constituida por dos representaciones originarias, del incondicionado y de lo condicionado. El conocimiento del primero es inmediato, e incluso la certeza que se tiene de su existencia es mayor que la que se tiene de la existencia de lo condicionado. Por otra parte, el conocimiento de este último tiene lugar de manera mediata, y en la medida en que se conoce el mecanismo mediante el cual el objeto se constituye como tal, nuestro entendimiento es capaz de reconstruirlo, y de esta reconstrucción proviene la certeza del conocimiento. Así, cuando la razón intenta encontrar las condiciones de posibilidad de lo incondicionado, procede de manera descabellada, ya que no hace más que exigir condiciones de algo que por definición no las tiene. Si fuera posible un concepto del incondicionado - esto es, de aquello que escapa a toda la red de vínculos que hacen posible que la razón construya la realidad natural—, éste dejaría de 
ser incondicionado, y debería entonces dejar de ser absolutamente necesario y comenzar a ser posible para dejarse construir, es decir, para poder ser concebido (Jacobi, 2000: 287-288).

Ahora bien, esto no significa que Jacobi niegue el incondicionado o que afirme que es imposible que el hombre lo conozca. Al contrario, nuestro filósofo reconoce que la naturaleza, la suma de lo condicionado, tiene lugar en el incondicionado, el cual, ya que se encuentra más allá de la naturaleza, debería ser considerado más bien como sobre-natural. En este sentido, si la naturaleza tuvo un inicio, este no ocurrió de manera natural, sino sobrenatural. Asimismo, la noción de inicio no es la misma que la razón concibe a partir de la naturaleza, esto es, de lo que está mediado naturalmente, sino que, por el contrario, solo puede reconocer una sola cosa con respecto a lo sobrenatural, que es un dato de hecho, a saber, que es. Este ser sobrenatural no tiene otro nombre que el de Dios, y no puede ser concebido como un simple arquitecto del universo, sino que antes que nada como creador, que no solo crea la sucesión de las cosas del mundo, sino que también la sustancia. De hecho, para Jacobi es tan ajeno a la operación de la razón el modo de ser de Dios, que considera irracional la exigencia de una demostración de Su existencia, y estima que la noción de una causa primera - deducida a partir del entendimiento y la voluntad del hombre- es imposible e insensata. Igualmente inaceptable sería lo que sostiene Spinoza, que Dios, dado que no puede ser corporal o un hombre, carece de individualidad y de intelecto (Jacobi, 2000: 288-290; Spinoza 2010: 44-48; Wolfson, 1933a: 311-319).

A este punto es evidente que para el filósofo de Düsseldorf la principal objeción contra la noción de sistema radica en que éste constituye el modo en que procede la razón cuando intenta aprehender - o, mejor dicho, abarcar- la realidad. De hecho, puesto que la razón considera real solo aquello que es capaz de construir de manera mediata, un objeto que se muestra de manera inmediata es inconcebible para ella, y por ende la razón no puede decidir su realidad ni tampoco afirmar su existencia efectiva, por mucho que lo considere como la realidad más alta. Por consiguiente, el sistema, el modo de proceder sistemático, constituye en último término el fundamento de la contradicción de la razón consigo misma, que le impide abrazar la existencia de Dios, de la cual estamos seguros porque Su presencia es inmediata, y no porque sea producto de una demostración (Jacobi, 2000: 290-291).

En respuesta a este dilema, nuestro filósofo encuentra un modo de actuar de la razón más allá del sistema, el cual se podría llamar el «puro instinto de la razón», y que ha movido desde siempre al hombre a considerar que la actividad espontánea de un ser vivo y autónomo precede a toda realidad. Ese modo de actuar es la voluntad. En efecto, sostiene Jacobi:

Sin embargo, nosotros poseemos — no obstante nuestra finitud y esclavitud naturales-, o al menos parece que poseemos en nosotros, a través de la conciencia de nuestra autonomía y espontaneidad [Selbsttätigkeit] en el ejercicio de nuestra voluntad, algo análogo a lo sobrenatural, esto es, del ser que no actúa de manera mecánica (Jacobi, 2000: 290). 
A partir de esto es posible ahora tener un punto de partida para apreciar la amplitud y profundidad de la discusión en torno al panteísmo. La crítica de Jacobi no se reduce a un mero examen de la doctrina de Spinoza con un interés simplemente académico, sino que mediante ella pretende poner en cuestión el modo de proceder mismo de la filosofía y el sentido en que la razón se considera capaz de decidir la realidad de lo real. Por ende, la contradicción del panteísmo y del creacionismo no radicaría tanto en lo que les es propio, sino en el hecho de que ambos son sistemas. Se podría decir entonces que el gran escándalo de la razón para el filósofo de Düsseldorf es el hecho que ella va de la mano con el sistema, ya que al proceder de esa manera se priva de la posibilidad de reconocer aquello que se manifiesta inmediatamente fuera de nosotros —esto es, el ser sobrenatural-, al tiempo que intenta porfiadamente remitir al sistema aquello que posee de inmediato y autónomo, a saber, la voluntad. Esta intuición es la que le mostrará el camino a Schelling. Para alcanzar el estadio de la libertad, se hace necesaria una discusión en torno al panteísmo - es decir, al determinismo, al mecanicismo, al fatalismo-, pero no solamente desde una posición que excluya del sistema toda posibilidad de conocer lo incondicionado y sobrenatural, sino desde un punto de vista que admita la posibilidad que el sistema se encuentre animado por el incondicionado, y que constituya un modo en que éste se pueda desplegar en la realidad. En otras palabras, en lugar de examinar la relación entre el sistema y Dios como un intento de aquél por forzar la presencia del incondicionado en el mecanismo, el filósofo de Leonberg intentará ver si se puede sostener el sentido contrario, que el incondicionado otorgue realidad al sistema, y si dicha afirmación no sería más adecuada al modo en que la realidad se manifiesta.

\section{La Respuesta de Schelling a Jacobi: los distintos SEnTidos del Panteísmo Y LA POSIBILIDAD DE UN SISTEMA DE LA LIBERTAD}

No hay que perder de vista que la cuestión fundamental, con la cual Schelling comienza su escrito sobre la libertad humana, consiste en la determinación de la posibilidad de un sistema de la libertad. Así, si bien dicho examen puede tener como resultado un concepto comprehensivo del fenómeno de la libertad, solo cuando se pone de manifiesto la relación que existe entre ese concepto y la totalidad de lo real y el modo en que se articula esa relación, el conocimiento que se tenga de la libertad alcanza su punto más alto, su última consumación científica. Esto es particularmente relevante en este caso, puesto que la libertad, como sostiene nuestro filósofo, «no debe ser un concepto simplemente subordinado o complementario, sino uno de los puntos centrales que dirigen el sistema» (Schelling, 2011: 9).

Es aquí también donde surge la polémica con Jacobi, ya que, como vimos, de acuerdo con él el sistema sería incompatible con una realidad que no se adecúa al mecanismo, y por lo tanto, como concluye Schelling, con la libertad, 
a tal punto que cualquier filosofía que pretenda reunir la totalidad y la unidad terminaría por negar la libertad. El filósofo de Leonberg señala diversos motivos por los cuales no se podría afirmar una incompatibilidad formal entre sistema y libertad, no obstante el hecho de que dicha incompatibilidad haya tenido efectivamente lugar a lo largo de la historia. Sin embargo, como sostiene Schelling, «la relación entre el concepto de libertad y la totalidad de la visión del mundo seguirá siendo siempre el objeto de una tarea necesaria, y hasta que no sea resuelta, el concepto mismo de libertad será vacilante, y la filosofía carecerá completamente de valor» (Schelling, 2011: 11). La tensión entre libertad y necesidad, que para Schelling constituye el hilo conductor que ha movido la filosofía a lo largo de la historia, debe ser considerada como la pregunta fundamental. Ahora bien, la forma que dicha pregunta ha adoptado en la época de nuestro filósofo, como vimos, es la de la controversia del panteísmo. Es por ello que una aclaración de esa cuestión se hace necesaria antes de examinar la libertad en cuanto tal. En consecuencia, Schelling se aboca a la tarea de elucidar los distintos sentidos en que ha sido interpretado el panteísmo en Spinoza.

\section{a. El examen de los distintos sentidos del panteísmo}

De acuerdo con nuestro filósofo, la noción de panteísmo se puede entender en primer lugar como una inmanencia en Dios, es decir, que todas las cosas se encuentran en Él. En este caso, Schelling observa que, si bien un fatalismo en este sentido podría tener lugar, ello no es prueba de que la inmanencia de las cosas en Dios conlleve esencialmente un fatalismo. Así, se puede afirmar que hay una contradicción entre libertad individual y casi todas las propiedades de un ser supremo, en particular con su omnipotencia. La libertad sería entonces un poder incondicionado, aparte y junto al poder divino, y por ende no se puede derivar a partir de este último, lo cual sería impensable, dado que ante la causalidad absoluta de un ser omnipotente a los demás seres no les queda más que una pasividad incondicionada. Por ese motivo, se podría pensar luego que la libertad no tendría cabida en este tipo de panteísmo. Incluso la afirmación de que, con el fin de salvaguardar la libertad, Dios podría retirarse, para así dar espacio a la acción libre del hombre, es un contrasentido que no soluciona nada, ya que si Dios dejara de actuar por un instante solo, el hombre dejaría inmediatamente de existir (Schelling, 2011: 11-12). Sin embargo, para nuestro filósofo precisamente el panteísmo entendido como inmanencia, como unidad de los hombres con Dios, da una cierta respuesta a dicha dificultad, ya que intenta salvar el actuar libre del hombre situándolo en Dios, y afirmando que habría una relación de pertenencia entre el actuar del hombre y la vida de Dios. Este es el sentido en que los místicos han creido que se alcanza una relación verdadera entre el hombre y Dios, y el sentimiento más profundo de unión con Dios les ha apetecido a ellos con igual o mayor fuerza que la razón y la especulación (Schelling, 2011: 12).

En segundo lugar, el panteísmo se puede entender en general como una identificación entre Dios y las cosas, esto es, mezclando al Creador con las 
criaturas. Sin embargo, Schelling hace notar que una interpretación del sistema de Spinoza que parte de dicha identificación no solo ignora la distinción absoluta (toto genere) entre Dios y las cosas planteada por el filósofo holandés, sino que hace dicha distinción casi impensable, ya que solo Dios es en sí y es el único ser que puede ser concebido a partir de sí mismo, mientras que las cosas finitas se encuentran necesariamente en otro y mediante éste pueden ser concebidas (Ibid.). Así, si se identifica a Dios con la totalidad de las cosas, resulta que el ser de estas últimas dependería absolutamente del ser de Dios, de tal manera que su relación sería casi como la que se da entre una premisa y sus consecuencias. En este sentido, dado que Dios es eterno, las cosas finitas también lo serían, pero no en sí, sino por derivación, es decir, como consecuencia de la existencia del ser infinito. Más aún, esta identificación se contradice a sí misma, puesto que la totalidad de las cosas no sería capaz de componer a Dios, en la medida en que constituyen algo posterior a él, del cual obtienen todo ser y toda determinación (Ibid.).

Otra manera de identificar a Dios con las cosas — que según Schelling es de peor gusto aún - interpreta la afirmación de Spinoza, de que cada cosa expresa la esencia de Dios de un modo cierto y determinado (Spinoza, 2010: 78), en el sentido de que cada cosa sería entonces igual a Dios. Para Schelling esto es inaceptable, puesto que un Dios derivado no es Dios en el sentido más propio, y por lo tanto la posición inicial se contradice, ya que resulta que las cosas están siempre separadas de Dios. El error radicaría aquí en el desconocimiento de la función del principio de identidad, o bien de la cópula en las proposiciones, en cuanto que una proposición que afirma que un cuerpo es azul, no quiere decir que el cuerpo en cuanto cuerpo se identifica con el azul, sino solo en relación con aquella parte del cuerpo que puede ser determinada por el color, de tal manera que al decir que un cuerpo es azul, se da por entendido que el cuerpo en primer lugar es cuerpo, pero también es azul (Schelling, 2011: 13-14). Así, por ejemplo, cuando se afirma que lo completo es lo incompleto, o que lo bueno es lo malo, eso no significa que haya una identificación absoluta entre sujeto y predicado, sino que se trata más bien de una remisión de lo que es negativo o carente a lo afirmativo o positivo. En este sentido, si la frase «lo bueno es lo malo» se entendiera como que ambos son uno y lo mismo, se podría negar la distinción entre justo e injusto, virtud y vicio, ya que entonces serían lógicamente idénticos. Incluso se podría llegar a afirmar, en este último sentido, que necesario y libre son idénticos, ya que un ser que es parte del mundo moral es también parte del mundo natural, y así la libertad sería una capacidad natural, un resorte, sometido como cualquier otra capacidad al mecanismo, o bien que el alma y el cuerpo, dado que son uno, serían idénticos, por lo que el alma sería algo corpóreo y el cuerpo algo espiritual (Schelling, 2011: 14). En este sentido, la distinción de la lógica antigua, que entiende sujeto y predicado como antecendente y consecuente, expresaría de manera adecuada el sentido real del principio de identidad, presente incluso en las proposiciones tautológicas, si es que se quiere afirmar algo mediante ellas. En efecto, cuando se dice que un cuerpo es un cuerpo, el sujeto expresa la unidad del concepto, mientras que el 
predicado nos remite a las propiedades particulares que se hallan contenidas en el concepto de cuerpo (Schelling, 2011: 15; Wulf, 2013: 135).

Siempre dentro de la interpretación que identifica a Dios con las cosas, nuestro filósofo distingue un tercer sentido, según el cual el problema del panteísmo no radica en que Dios sea la totalidad de las cosas, sino que más bien en el hecho de que las cosas en sí no serían nada, y por ende este sistema anularía toda individualidad. Aquí se puede observar inmediatamente una contradicción, ya que sería imposible mezclar a Dios, que es, con las cosas, que no son. En ese mismo sentido, si no hay nada aparte de Dios - esto es, tanto extra como praeter Deum-, no se podría decir más que como mera palabrería que Dios es todas las cosas (Schelling, 2011: 16). Incluso es dudoso que esta interpretación se aplique a Spinoza, ya que cuando reconoce que aparte (praeter) de la sustancia solo se dan sus afecciones, que permiten explicar las cosas (Spinoza 2010: 56. 118), esta noción es negativa, y no hay algo positivo o esencial respecto a las cosas en ella, sino que solo sirve para determinar su relación con Dios. Lo que Spinoza estaría afirmando, sería más bien que cada cosa consiste en la sustancia, considerada según uno de sus modos. En efecto, si se considera la sustancia con uno de sus atributos, lo positivo es siempre la sustancia, pero de eso no se concluye que la sustancia considerada con sus infinitos atributos (A/a $\mathrm{A} / \mathrm{b}, \mathrm{A} / \mathrm{c}$, etc.) sea idéntica a la sustancia por sí sola (A), o más bien que la sustancia y su atributo no puedan ser una cosa determinada, a pesar de que sea una consecuencia de la substancia. Tal afirmación, sin embargo, no se encuentra en Spinoza (Schelling, 2011: 17).

Finalmente, el filósofo de Leonberg identifica un tercer sentido general del panteísmo, según el cual éste sería una negación ya no de la individualidad, sino de la libertad. En este caso, sin embargo, serían varios los sistemas, y no solo el espinosismo, los que podrían ser considerados panteístas. De hecho, hasta el descubrimiento del Idealismo, el concepto propiamente tal de libertad habría estado ausente de los sistemas filosóficos en general, tanto del de Leibniz como del de Spinoza, e incluso la noción de libertad que el mismo Jacobi propone se podría deducir con facilidad de Spinoza. Es por ello que Schelling considera que la afirmación o la negación de la libertad no tendría que ver en primer lugar con la negación o la afirmación del panteísmo —entendido en cuanto inmanencia de las cosas en Dios-, pues la contraposición entre libertad y omnipotencia divina provendría de una comprensión errada del principio de identidad, y la unidad de este principio no es estática, sino más bien creadora. En este sentido, al referirse a la relación entre sujeto y predicado, nuestro filósofo aludió a la relación entre fundamento (Grund) y consecuencia, y se puede afirmar que el principio de razón (Grund) es tan originario como el de identidad. Así, lo eterno debe ser de manera inmediata y, en cuanto que está en sí mismo, fundamento; al mismo tiempo, es también derivado, en la medida en que constituye el fundamento de sí mismo, y se concibe a partir de sí mismo, en virtud de su inmanencia en sí. Ahora bien, la dependencia se opone a la independencia, no a la libertad, ya que no determina la esencia de aquello que está presente en otro, sino que solo quiere decir que aquello que está en 
otro depende de este último. En efecto, la dependencia en los seres orgánicos dice relación con el hecho de que uno depende del otro en cuanto a su venir a ser, no en cuanto a su ser tal o cual. Así, no hay contradicción si el hijo de un hombre es también un hombre; contradictorio sería si lo dependiente no fuera a su vez autónomo, ya que se trataría de una dependencia sin dependiente, de una consecuencia sin consecuente. Otro tanto ocurre con lo que se concibe en otro, como las partes del cuerpo, que por sí solas no pueden subsistir, y que al mismo tiempo poseen una cierta independencia respecto de todo el cuerpo (Schelling, 2011: 18-19).

Ahora bien, si se considera cuál podría ser la relación entre el ser divino y aquello que es producido por él, la interpretación negativa del panteísmo termina de caer por su propio peso. Al respecto, Schelling afirma que una consecuencia de la esencia divina que no fuera a su vez una procreación, esto es, que no diera lugar a un ser autónomo, se contradice a sí misma. Dios no es un Dios de los muertos, dice el Evangelio (Lc. 20, 37-38), sino de los vivos, y por ello no se entiende cómo el ser más perfecto en absoluto podría tener deseo de estar en la máquina más perfecta posible. En este sentido, sea como sea que se pensara la derivación de un ser a partir de Dios, no podría tener lugar como algo mecánico, como una mera producción o disposición, tampoco como una emanación en donde lo que surge permaneciera en aquello de lo cual surgió, sin poseer nada propio o autónomo. Por el contrario, la proveniencia de las cosas a partir de Dios constituye más bien para Schelling una revelación de Dios a partir de sí mismo y, dado que «solo se puede revelar en aquello que es similar a él, [se revela] a cosas que pueden actuar de manera libre. No hay otro fundamento para el ser de estas últimas más que Dios, las cuales son tal cual Dios es. Él habla, y las cosas tienen lugar» (Schelling, 2011: 19). Incluso si los seres del mundo fueran simples pensamientos de Dios, y por ende tuvieran lugar en el alma divina solamente, aún así poseerían su propia independencia, como lo demuestran los pensamientos que genera el intelecto humano, cuya fuerza es tan avasalladora, que lo dominan y subyugan (ibid.). Por otra parte, a diferencia del intelecto humano, que solo puede otorgar a sus conceptos una realidad ideal, las representaciones del intelecto divino deberían ser lisa y llanamente seres autónomos, en la medida en que:

Dios contempla las cosas en sí. En sí es solamente lo eterno, lo que reside en sí mismo, la voluntad, la libertad. El concepto de un absoluto derivado o de una divinidad [derivada] es tan poco contradictorio, que es más bien el término medio de toda la filosofía. Una divinidad tal está en correspondencia con la naturaleza, se aviene a ella [kommt der Natur zu]. Tan poco contradictorias son la inmanencia en Dios y la libertad, que precisamente solo lo libre, y hasta el punto en que es libre, está en Dios, y lo no libre, y hasta el punto en que no es libre, está necesariamente fuera de Dios (Schelling, 2011: 20).

Con estas palabras Schelling concluye su examen del panteísmo, y con ellas vuelve al primer sentido en que había entendido dicha noción, a saber, como una inmanencia de las cosas en Dios, mostrando el sentido positivo de dicha 
inmanencia, ya que, en vez de negar la libertad, el hecho de que las cosas estén en Dios la confirma y le proporciona un lugar seguro, donde no corre el riesgo de ser sometida a un sistema mecánico, determinista, sino más bien donde se preserva y puede revelar su carácter incondicionado (Wulf, 2013: 131-132). Sin embargo, como se vio recién, dicho estar en Dios no consiste en un simple hallarse, como se hallan los distintos engranajes en un reloj, sino que más bien aquello que está en Dios, está en él porque es capaz de acoger su manifestación, ya no porque posea un intelecto capaz de reconocer la presencia del ser divino, sino porque es libre. La libertad es la impronta de la divinidad. Este es el punto que le será útil a Schelling para discutir la posición de Spinoza, pero también la crítica de Jacobi a la noción de sistema.

\section{b. La crítica al espinosismo}

Ahora bien, si de acuerdo con Schelling una negación de la libertad formal no está necesariamente vinculada con el panteísmo — sino que incluso solo aquello que es libre puede tener lugar en Dios-, queda aún otro aspecto de la crítica a Spinoza por examinar, a saber, que el espinosismo constituiría el sistema de razón por antonomasia $\left(\varkappa \alpha ̀ \tau^{\prime} \dot{\varepsilon} \xi o \chi \eta^{\prime} v\right)$, aquel que tendría lugar en la razón de todo ser humano. Nuestro filósofo se pregunta, entonces, qué se entiende por espinosismo, si se trata de toda su doctrina - tanto física como metafísica-, o bien de una parte de ella, sin que se diga sobre qué principio se decide qué se admite como consecuencia de dicho principio y qué se desecha. $\mathrm{Al}$ respecto, el filósofo de Leonberg sostiene que cada cierto tiempo en la historia del desarrollo del espíritu alemán tiene lugar un fenómeno interesante, a saber, que se afirma que el sistema que identifica a Dios con las cosas, y que somete todo a un mecanismo ciegamente necesario, es el único sistema que la razón pura es capaz de desarrollar. Según Schelling, este modo de pensar es propio del espíritu de una época pasada, que encontró en el ateísmo francés su punto culminante, y que también se quiso implantar en Alemania. Sin embargo, dado que ese espíritu no era totalmente compatible con la mentalidad alemana, se comenzó a difundir la separación entre mente y corazón, según la cual se aborrecen las conclusiones, sin que en el fondo se liberen del modo de pensar a partir del cual surgieron, o se intente proponer uno mejor. Así, no hubo más remedio que afirmar la terrible verdad, a saber, que toda filosofía, en particular aquella que es puramente racional, es o terminará en espinosismo. Una vez que se presentó a los alemanes el abismo que se abría ante sus pies, se tomó la medida más audaz, de reconducir la filosofía al corazón, al sentimiento y a la fe. Schelling ve con alivio que este modo de pensar ya quedó en el pasado, en la medida en que su época estaba iluminada por la luz el Idealismo, de modo que la identificación entre sistema y razón no podría ser afirmada en el mismo sentido ni conduciría a las mismas conclusiones (Schelling, 2011: 20-21).

Es aquí donde nuestro filósofo considera necesario dar su propio parecer en relación con el espinosismo. En primer lugar, el panteísmo no es fatalismo porque afirme que todas las cosas están en Dios, ya que, como el mismo Schelling 
ha intentado demostrar, la inmanencia no hace imposible la libertad —al menos en sentido formal - sino que, por el contrario, exige que los seres que están en el ser absolutamente libre sean ellos a su vez libres. Por lo tanto, si se puede decir que Spinoza es fatalista, esto no provendría del solo hecho de que sostenga que las cosas estén en Dios. La falla de su sistema, entonces, no radicaría en dicha inmanencia, sino en el hecho de que siempre se trata de cosas - entendiendo «cosa» en el sentido abstracto en que lo son los seres naturales-, y que tanto la sustancia como los seres derivados de ella serían en cada caso cosas. Por consiguiente, sus argumentos contra la libertad serían más bien deterministas, de ningún modo panteístas. Más aún, trata como una cosa incluso a la voluntad misma, y entiende su actuar en sentido naturalista, en la medida en que una acción siempre depende de otra cosa para actuar, y esta última, a su vez, de la acción de otra más, y así hasta el infinito. Es por eso que su sistema - y la exánime, seca exposición de éste- carece de vida, y que su visión de la naturaleza es puramente mecánica (Schelling, 2011: 21-22).

Todo esto debería cambiar, sin embargo, si se considera a la naturaleza de una manera dinámica, viva. En este sentido, un sistema cuyo fundamento consiste en que todas las cosas sean concebidas en Dios debería ser animado y purgado de toda abstracción para poder constituirse en el principio fundamental de un sistema racional. Schelling compara el sistema de Spinoza a las estatuas de Pigmalión, que adquirían vida mediante un soplo de amor, con la única diferencia que, si cobrara vida, el sistema del filósofo holandés pondría en evidencia con mayor claridad su carácter inacabado, similar a las representaciones antiguas de las divinidades, que cuanto más incompletas fueran y más carecieran de rasgos particulares, tanto más misteriosas aparecían (Schelling, 2011: 22).

En conclusión, afirma Schelling que, si hubiera que llamar de algúna modo al sistema de Spinoza, el nombre más adecuado sería el de un sistema realista unilateral. Éste implica una condena mucho menor que el de panteísmo, y al mismo tiempo describe mejor aquello que le es propio. En este punto, nuestro filósofo se remite a sus propios escritos, para poner en evidencia la influencia que ha tenido el espinosismo sobre su propio pensamiento, así como también para dejar claro que el principio a partir del cual surge dicho pensamiento es radicalmente distinto (Wulf, 2013: 139-143). En efecto, como él mismo reconoce, la intención expresa de sus esfuerzos especulativos ha sido hacer que se permeen, que se penetren recíprocamente el Realismo y el Idealismo (Schelling, 2011: 22-23). Y es sobre esta base que el párrafo citado al comienzo de este trabajo nos permite apreciar mejor el alcance de todo este examen:

El concepto fundamental de Spinoza, espiritualizado por el principio del Idealismo (y transformado en un punto esencial), alcanzó en un modo superior de consideración de la naturaleza, y en la reconocida unidad de lo dinámico con lo anímico y lo espiritual, una base viva, a partir de la cual surgió una Filosofía de la Naturaleza que podría subsistir por sí misma en tanto que mera física, pero que en relación con la totalidad de la filosofía fue considerada en todo tiempo como una sola parte de ella, en concreto, como la parte 
real, que solo entonces se torna capaz de elevarse hasta alcanzar el auténtico sistema de razón, añadiéndole la parte ideal, en la que reina la libertad. Se afirma que en ella (en la libertad) reside el último acto potenciador, gracias al cual toda naturaleza se transfigura en sensación, en inteligencia y, finalmente, en voluntad. - En la más alta y última instancia, no hay otro ser sino querer. Querer es el ser originario [Ursein] y solo en él concuerdan todos los predicados de este último: ausencia de fundamento, eternidad, independencia respecto al tiempo, autoafirmación. Toda la filosofía pugna solo por esto, por encontrar esta expresión suprema (Schelling, 2011: 23).

Tanto la composición de este párrafo, como la línea de argumentación que ha seguido Schelling hasta este momento, dan como resultado que el esfuerzo especulativo de la reflexión sobre el panteísmo - y se podría decir, tanto en lo que se refiere al examen de nuestro filósofo en su escrito, como la discusión que tuvo lugar desde la época de la "polémica del panteísmo»-confluye en la sentencia "Querer es el ser originario». Y si Kant concibe la voluntad como el principio incondicionado que tiene lugar en el sujeto, que debe recorrer el camino inverso a la razón especulativa, y pasar de la razón pura a la experiencia, Schelling da el paso sucesivo y ubica la voluntad más allá del sujeto, como principio rector que anima toda la realidad, y en particular como aquello que decide la relación del ser supremo con los demás seres. A continuación se intentará mostrar algunas consecuencias especulativas de esta afirmación.

\section{CONSIDERACIONES A MODO DE CONCLUSIÓN}

La discusión sobre el panteísmo, que a primera vista podría parecer una cuestión que posee un interés meramente histórico o académico, muestra su verdadero sentido y alcance en la afirmación del carácter originario de la voluntad. Schelling es consciente de las implicancias de una afirmación tal, y no se puede evitar percibir un dejo de orgullo — de ninguna manera vano, sino que incluso bien merecido- cuando sostiene que toda la filosofía pugna por alcanzar dicha expresión suprema que él ha formulado. Dicho orgullo se podría atribuir, antes que a la magnitud del resultado al que ha llegado nuestro filósofo, más bien al hecho de que con ello se ha insertado en la tradición filosófica que comienza con Descartes, y que encuentra en Spinoza, Leibniz y Kant a algunos de sus exponentes más notables. En efecto, al poner a la voluntad como primer principio, por encima de la especulación —es decir, por encima del sistema en sentido mecanicista, determinista, fatalista- y sobre el mero ser - esto es, sobre el simple «estar ahí» de las cosas que no poseen conciencia de sí mismas, y que por ende se hallan irremediablemente sometidas al sistema-, Schelling deja al descubierto el principio que ha animado el pensamiento moderno, mientras que en la discusión sobre el panteísmo resume la evolución que ha tenido la conciencia que dicha tradición ha ido adquiriendo sobre sus propios principios especulativos. Incluso es posible advertir cómo nuestro filósofo, cuando al final de este tratado distingue dos tipos de seres - por una 
parte el hombre, quien, en cuanto ser dotado de libertad, es un ser central, que se encuentra en el centro que es Dios, y los seres meramente naturales, cuya existencia es en sí "periférica», pero que por intermedio del hombre pueden ser acogidos por Dios y vinculados a él (Schelling, 2013: 82-83)—, lleva a cabo un camino similar al de Descartes, cuando se sirve de la noción de infinitud para mostrar cómo es que la res cogitans, que hasta ese punto de la argumentación habría sido capaz de dar un fundamento real —o bien, en palabras de Descartes, «realidad objetiva» - a todas las representaciones, toma conciencia de que sus representaciones en último término provienen de algo que la supera, que se encuentra más allá de la cogitatio, y que por ende constituye el fundamento real de las distintas cogitationes y del ser en el que ellas tienen lugar (Descartes, 1983: 40-52). De esta manera, se podría afirmar que Schelling lleva a cabo una reflexión esencialmente moderna sobre la modernidad misma.

Esta meditación, si bien interpela a aquello que se puede considerar como lo más propio del pensar moderno, a saber, que su forma no es otra que el sistema, no pretende superar el sistema, sino que más bien intenta superar su carácter meramente mecanicista y hallar aquello que anima al sistema y a la realidad. En este sentido, la crítica de Schelling apunta tanto a las interpretaciones que consideran el espinosismo como panteísmo, como al mismo Spinoza. A partir de ese examen queda claro que no se puede decir que nuestro filósofo esté totalmente de acuerdo con Spinoza, al contrario, reconoce y destaca sus numerosas limitaciones y carencias. Sin embargo, los defectos de ese sistema solo pueden quedar en evidencia si se le compara con una versión mejor del sistema, que logre dar cuenta de la necesidad al interior de él y asimismo de la libertad que en último término constituye aquello que le da lugar y que lo anima (Wulf, 2013: 143-145).

Ahora bien, se puede afirmar que el carácter moderno de dicha meditación viene dado por la discusión con Jacobi, en la medida en que el problema del panteísmo se puede entender como una discusión histórica. En efecto, la importancia del apéndice VII al texto sobre Spinoza radica precisamente en el hecho de que los argumentos de Jacobi contra el filósofo holandés y contra el sistema en general pasan a ser una clave de lectura de la historia de la filosofía, la cual se concibe entonces como una sucesión de sistemas y con ello, en cierta medida, como la crónica del fracaso de la razón humana en sus intentos por comprender la realidad, por su deseo de asirla y confinarla en un sistema. Como afirmará años después en sus cursos sobre la filosofía moderna, Schelling advierte en Jacobi la influencia de Pascal, a quien nuestro filósofo considera el modelo a seguir para quienes quieren saber en qué consiste un sistema histórico, es decir, un sistema que es capaz de acoger en sí aquello vivo que permanece de la historia (Schelling, 2013: 120). Es posible concluir, por ende, que el sentido histórico en que se debería entender la polémica del panteísmo, a partir de la discusión de y con Jacobi, ya no se reduce a una controversia contingente, sino a un problema que atañe a la historia de la filosofía en su conjunto, entendida entonces como historia de los distintos modos en que el pensamiento humano se ha desplegado a lo largo de las generaciones. 
Precisamente en relación a dichos modos en que se ha desplegado el pensamiento en las distintas épocas, es que la discusión con Jacobi adquiere un aspecto metafísico, que exige un examen más detallado y más fino que el que se puede llevar a cabo en estas breves reflexiones conclusivas. Baste, por ahora, con que se enuncie. La diferencia radical entre Schelling y Jacobi atañe en último término a la comprensión que cada uno de ellos posee de la noción de ser. En efecto, para el filósofo de Düsseldorf, la manifestación de aquello vivo, que se muestra a partir de sí mismo, y que por ende no puede ser conocido a partir de otro, es incompatible con la razón que solo puede conocer reconociéndose a sí misma como fundamento del conocimiento. Es por ello que Jacobi apela al sentimiento para afirmar que el reconocimiento de la presencia de lo sobrenatural es tanto o más evidente que la de lo natural, ya que se da inmediatamente, y no requiere la mediación de la demostración (Jacobi, 2000: 316). Así, en la medida en que nuestro autor separa aquello que concibe la manifestación de lo natural de lo que siente la presencia de lo sobrenatural, separa también el modo de ser de ambos ámbitos, y con ello separa a su vez la razón del hombre de su corazón (Pascal, 1909: 458). Schelling, en cambio, considera que es el hombre aquel que sirve como mediador entre el ser sobrenatural y los seres naturales, no porque conoce, sino porque quiere. El querer, el incondicionado que se reconoce como ya presente en la razón, en el que concuerdan los predicados del ser original, es el punto en el que confluyen Dios, el ser que es el centro de toda realidad, y el hombre, que es el único ser central, en la medida en que posee una «nostalgia inicial», que lo lleva a buscar la unidad entre su devenir y su entendimiento (Schelling, 2011: 82). En torno a dicho centro, que comparte un modo de ser común, se difunde el ser a las criaturas, que hasta que no son animadas e interpretadas por el hombre, poseen, como ya se dijo, un ser meramente periférico. La interpretación de la realidad que lleva a cabo el hombre, entonces, aún cuando tome la forma de un sistema, por el hecho de acercar a la naturaleza a Dios, constituye algo vivo y vivificante. El sistema, en último término, es libre, ya que la libertad es su fundamento. Queda, sin embargo, pendiente la decisión acerca de si el sistema constituye la forma intrínseca de la filosofía, es decir, si solo en el sistema es posible hablar de filosofía o no.

\section{Bibliografía}

BeIser, F. C. (1987) The Fate of Reason. German Philosophy from Kant to Fichte. Cambridge, MA: Harvard University Press.

Brown, J. V. (1976) «John Duns Scotus on Henry of Ghent's Arguments for Divine Illumination: The Statement of the Case». Vivarium 14, 94-113.

- (1984) «Duns Scotus on the Possibility of Knowing Genuine Truth: The Reply to Henry of Ghent in the Lectura prima and the Ordinatio». Recherches de théologie ancienne et médiévale 51, 136-182.

Carrasco Conde, A. (2011) «Panteísmo y panenteísmo: Schelling, Schlegel y la polémica

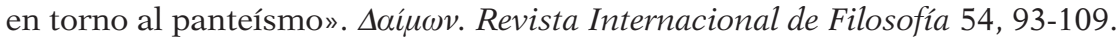


Descartes, R. (1982) Principia Philosophiae. En: Oeuvres de Descartes, ed. Ch. Adam - P. Tannery, nouvelle présentation, en coédition avec le CNRS, vol. $8^{1}$, Paris: Vrin.

- (1983) Meditationes de Prima Philosophia. En: Oeuvres, vol 7.

Duns Scoto, J. (1950) Ordinatio prologus. En: B. Ioannis Duns Scoti Opera omnia, ed. Commissio Scotista, vol. 1. Civitas Vaticana: Typis Polyglottis Vaticanis.

- (1954) Ordinatio I, d. 3. En Opera omnia, vol. 3.

Freudiger, J. (1993) «Der Pantheismusstreit - Eine Bestandsaufnahme». Kriterion 5, 39-48.

Guerrero Troncoso, H. (2016) «Ley, naturaleza y gracia. Una lectura escotista de la ley natural en santo Tomás». En: Casanova, C. - Serrano, I. (eds.) Gratia non tollit naturam, sed perficit eam. Sobre las relaciones y límites entre naturaleza y gracia. Actas del Segundo Congreso Internacional de Filosofía Tomista. Santiago: Universidad Santo Tomás - RIL Editores, 413-440.

Hegel, G. W. F. (1988) Phänomenologie des Geistes. Ed. H.-F. Wessels-H. Clairmont. Hamburg: Meiner.

JAcoвI, F. H. (2000) Über die Lehre des Spinoza in Briefe an Herrn Moses Mendelssohn. Ed. LauschKe, M. Hamburg: Meiner.

JAKUSzKo, H. (2009) "The Spinoza Inspiration in the Late German Enlightenment (Spätaufklärung)». Studies in Logic, Grammar and Rhetoric 15 (28), 173-188.

Kahlefeld, S. (2000) Dialektik und Sprung in Jacobis Philosophie. Würzburg: Königshausen \& Neumann.

Kant, I. (1998) Kritik der reinen Vernunft. Ed. Timmermann, J. Hamburg: Meiner.

Pascal, B. (1909) Penseés et opuscules. Ed. Brunschvicg, L., 5a edición. Paris: Hachette.

Schelling, F. W. J. (2011) Philosophische Untersuchungen über das Wesen der menschlichen Freiheit und die damit zusammenhängende Gegenstände. Ed. Buсннеім, Th. Hamburg: Meiner.

- (2013) Zur Geschichte der neueren Philosophie. Münchener Vorlesungen. Ed. HolzINGER, M. Berlin: Holzinger.

Spinoza, B. (2010) Ethica ordine geometrico demonstrata. Ed. Bartuschat, W. Hamburg: Meiner.

Wolf, S. (2013) «Pantheismus und Pantheismuskritik in Schellings Freiheitsschrift». SATS. Northern European Journal of Philosophy 132, 128-146.

Wolfson, H. A. (1934a-1934b) The Philosophy of Spinoza. Unfolding the Latent Processes of his Reasoning, 2 vols. Cambridge, MA: Harvard University Press.

Universidad Católica del Maule

Facultad de Ciencias Religiosas y Filosóficas

Hernán Guerrero Troncoso

Avda. San Miguel 3605

Talca, VII Región, Chile

herrguerrero@gmail.com

[Artículo aprobado para publicación en diciembre de 2015] 